\title{
L'ÉLEVAGE DE LA TRUITE FARIO ET DE LA TRUITE ARC-EN-CIEL DANS LES EAUX A TEMPÉRATURE TRÈS ELEVÉE ${ }^{(1)}$
}

\author{
par ENrique G. CALDERON \\ Chef de la Station Centrale d'Hydrobiologie \\ Service national de péche fluviale et chasse, Madrid (Espagne).
}

\section{BUTS}

Un cours d'eau se divise en diverses zones de sa source dans les montagnes à son embouchure dans la mer. La Truite habite la première de ces zones caractérisée par de basses températures inférieures à $22^{\circ} \mathrm{C}$, des eaux rapides et fortement oxygénées qui correspondent à un lit à forte pente et de faible largeur moyenne.

Tout ceci explique que les piscicultures de salmonidés sont situées seulement dans les zones montagneuses et c'est pourquoi dans tous les pays elles se trouvent centralisées dans des régions très caractéristiques.

La croissance rapide de la Truite arc-en-ciel et sa grande facilité d'acclimatation dans des eaux présentant des conditions différentes de celles où elle habita naturellement ont fait qu'en 1960 nous avons commencé une série d'études sur cette espèce afin de tenter d'étendre son aire d'élevage. Les résultats favorables que nous avons obtenus ont amené à effectuer aussi des expériences avec la Truite commune qui, bien qu'en plus faible proportion (parce que nous ne disposions pas d'un nombre suffisant d'étangs pour l'expérimentation), nous a cependant permis d'obtenir quelques résultats et de déduire les conséquences qui en découlent.

Le problème essentiel dans l'élevage de Truites dans les eaux à température élevée est que cette espèce rencontre des difficultés à vivre dans des eaux à une température dépassant $22^{\circ} \mathrm{C}$. Cette limite de température provient $d u$ fait qu'il existe une corrélation entre la température de l'eau et la tension d'oxygène dissous et qu'à $22^{\circ}$ correspond une tension de $8,83 \mathrm{mg}$ d'O $\mathrm{O}_{2}$ par litre ce qui est la quantité normale nécessaire à l'espèce.

(1) Extrait d'Études et Revues, nº 30, décembre 1965. Conseil Général des Pêches pour la Méditerranée (C. G. P. M.), Organisation des Nations Unies pour l'Alimentation et l'Agriculture (F. A. O.), Rome, et publié avec l'aimable autorisation de l'Auteur. 
Pour cela, nos expériences se sont déroulées à partir d'un principe : obtenir d'une manière naturelle une sursaturation de l'eau en oxygène et éviter ainsi la nécessité que cette eau soit à de basses températures.

On a effectué des études des caractéristiques de l'incubation (température, degrés-jours nécessaires pour atteindre les phases distinctes, etc.) du développement (poids et longueurs atteints aux âges distincts) et caractéristiques de l'alevinage (température, oxygénation de l'eau, végétation, etc.).

\section{MATÉRIEL ET MÉTHODES}

\section{1. Garactéristiques de l'établissement de pisciculture.}

Les travaux ont été réalisés à la pisciculture expérimentale d'Aranjuez (Madrid), du Service national de la pêche fluviale et de la chasse.

Cette pisciculture est située près du fleuve Tajo et dans les jardins du prince d'Aranjuez, situation géographique : $40^{\circ} 01^{\prime} \mathrm{N}, 3^{\circ} 43^{\prime} \mathrm{W}$, altitude $489 \mathrm{~m}$. L'eau utilisée provient du fleuve Tajo dont la zone piscicole dans cette section correspond à la transition entre la "zone à barbeau " et la "zone à carpe ".

Les étangs occupent une surface de $5.207 \mathrm{~m}^{2}$ dont $2.355 \mathrm{~m}^{2}$ comprennent 25 étangs de béton et $2.852 \mathrm{~m}^{2}$, 10 étangs de terre. Dans les étangs de béton pour l'élevage de la Truite on a disposé sur une partie du fond de la terre végétale garnie de végétation aquatique. La hauteur moyenne de l'eau dans les étangs est de quelque $80 \mathrm{~cm}$. L'eau utilisée pour l'alimentation des étangs vient directement du fleuve Tajo, par un canal général de $280 \mathrm{~m}$ de long et est pompée par des groupes motopompes et uniquement quand l'eau doit alimenter le laboratoire où se font l'incubation et la production d'alevins, elle est filtrée à travers un filtre de gravier et de sable constitué par deux bassins de $12 \mathrm{~m}^{2}$ de surface.

Le débit d'eau dans les bassins, étangs et laboratoire d'incubation est de $45.000 \mathrm{l} / \mathrm{h}$, celui correspondant aux bassins étant variable selon les expériences qui y sont effectuées, mais on peut l'évaluer en moyenne à 0,21 par minute et par mètre carré de surface de bassin.

L'incubation des œufs se fait dans des bacs de béton de $2,27 \times$ $0,49 \times 0,45 \mathrm{~m}$, munis des plateaux appropriés et, après l'éclosion, les jeunes alevins restent dans les bacs pendant un premier stade.

\section{2. Provenance des individus.}

En 1958, à la Pisciculture du Monastère de Piedra (Alhama de Aragon, Saragosse), typique pour ses eaux froides, on a effectué comme tous les ans la ponte artificielle des Truites et une partie des œufs fécondés a été transportée le jour même de la fécondation à la pisciculture d'Aranjuez afin d'étudier la possibilité de son élevage, car cette espèce n'y avait jamais été élevée.

L'incubation de ces œufs a été faite dans cet établissement dans de l'eau à température élevée, et elle a donné des reproducteurs qui, en 1960, 
ont permis par fécondation artificielle d'obtenir les Truites avec lesquelles les travaux que nous rapportons ici ont pu être réalisés.

Par conséquent, tous les exemplaires soumis aux expériences sont nés à la pisciculture d'Aranjuez et proviennent de reproducteurs qui y sont nés et qui ont été à l'origine de plusieurs générations de Truites lesquelles, à leur tour, se sont reproduites et leurs descendants se sont mélangés aux individus étudiés.

\section{3. Nourriture.}

Pendant l'alevinage, l'aliment fourni a été de la rate de bœuf avec un complément de plancton provenant des bassins. Plus tard, l'aliment des alevins et des Truites était composé d'un mélange de viandes (cheval ou bœuf), de farine de poisson, de son et d'un complément de vitamines. La quantité donnée dans les bassins était celle nécessaire pour satisfaire les besoins des individus qui s'y trouvaient et, dans quelques cas, pour compléter cette nourriture artificielle on a fait un déversement périodique de gambusia (Gambusia holbrocki) pour qu'il y en ait toujours dans les bassins à la disposition des Truites.

A partir de mars 1963, on a essayé une nourriture artificielle granulée dont nous donnons plus loin la composition et qui a remplacé le mélange de farine de poisson, viande, etc., mais les résultats de ses effets sur l'élevage que nous faisons actuellement ne sont pas déterminés bien que jusqu'à présent cette nourriture paraisse affecter défavorablement la faculté de reproduction des adultes.

La composition de cet aliment est la suivante :

\begin{tabular}{|c|c|}
\hline "Tercerillas $"$ de blé $\ldots . . \ldots \ldots \ldots$ & $22 \%$ \\
\hline Farine de pois blanc. $\ldots \ldots \ldots \ldots$ & $38 \%$ \\
\hline Farine de graines de coton .... & $15 \%$ \\
\hline Lait écrémé en poudre . . . & $5,5 \%$ \\
\hline Farine d'os $\ldots \ldots \ldots$ & $5 \%$ \\
\hline Levure de bière en poudre...... & $10 \%$ \\
\hline Mélange de vitamines $\ldots \ldots \ldots \ldots$ & $1,5 \%$ \\
\hline Huile comestible A-D .. & $3 \%$ \\
\hline Eau ajoutée à $100 \mathrm{~g}$ de mélange. & $6 \mathrm{~cm}^{3}$ \\
\hline
\end{tabular}

\section{4. Contrôle.}

La fécondation artificielle pendant les travaux a été effectuée selon la méthode à sec, l'incubation des cufs se faisant sur des plateaux appropriés. Pour le contrôle du déroulement de l'incubation et de l'alevinage on a utilisé des fiches spéciales.

Pour la détermination de la croissance, on a utilisé pour les individus vivants une série de relèvements de la longueur, en utilisant pour cela des règles de plastique spéciales divisées en centimètre sur lesquelles on plaçait les individus pendant quelques secondes. Immédiatement après, les mêmes individus étaient posés pendant quelques secondes 
sur du papier filtre sec, pour éliminer la majeure partie de l'eau qu'ils pouvaient entraîner, et on les plaçait ensuite dans un matras avec de l'eau bien oxygénée pour les peser.

Le poids a été déterminé par la différence entre la tare et le poids brut, au décigramme pour les petites Truites, au demi-gramme pour celles de 50 à $100 \mathrm{~g}$, et au gramme quand il s'agissait de grands exemplaires.

\section{EXPERIENCES ET RÉSULTATS}

\section{1. Caractéristiques de l'eau d'élevage.}

\section{I. 1. - Analyse chimique.}

L'analyse chimique de l'eau d'alimentation des bassins a donné les résultats suivants :

\begin{tabular}{|c|c|}
\hline$p \mathrm{H} \ldots$ & 6,2 \\
\hline SBV . . . . . . & 3,20 \\
\hline Dureté totale $\ldots$ & $50^{\circ} \mathrm{Fr}$ \\
\hline Matière organique & 1,68 ppm. O \\
\hline Calcium.............. & $164 \mathrm{ppm} . \mathrm{Ca}$ \\
\hline Chlorures . . . . . . . . & 42,5 ppm. C1 \\
\hline Sulfates ........... & 346 ppm. $\mathrm{SO}_{4}$ \\
\hline Nitrates $\ldots \ldots \ldots \ldots \ldots$ & Traces \\
\hline Nitrites $\ldots .$. & $"$ \\
\hline Ammonium & \\
\hline
\end{tabular}

\section{1. 2. - Turbidité.}

La turbidité de l'eau a été mesurée tous les 3 jours avec un népholomètre Lange. Les résultats obtenus figurent dans le graphique $n^{\circ} 1$, et il y apparaît que les maximums correspondaient à février-mars et à juin-juillet.

On a fait le graphique des valeurs correspondant à la turbidité de l'eau dans le canal d'entrée pour l'alimentation de la pisciculture et de celle de l'eau des bassins. En général, ces valeurs sont assez voisines mais quand il se produit une grande élévation de la turbidité de l'eau elle est amortie dans le bassin - ce qui est dû à la faible quantité d'eau qui y circule et à la décantation correspondante des matières en suspension qui s'y produit.

\section{1. 3. - Oxygène dissous.}

Comme nous l'avons dit auparavant, notre but pour résoudre le problème de l'élevage des Truites dans des eaux à température élevée, a été de procéder d'une manière naturelle à une sursaturation de l'eau en oxygène dissous. Nous l'avons obtenu par l'intervention de certains facteurs, tels que la végétation submergée, l'ensoleillement et le débit d'eau.

La végétation employéc comprenait Potamogeton crispus, Ceratophyllum demersum, Anacharis (Elodea) canadensis. La surface portant 


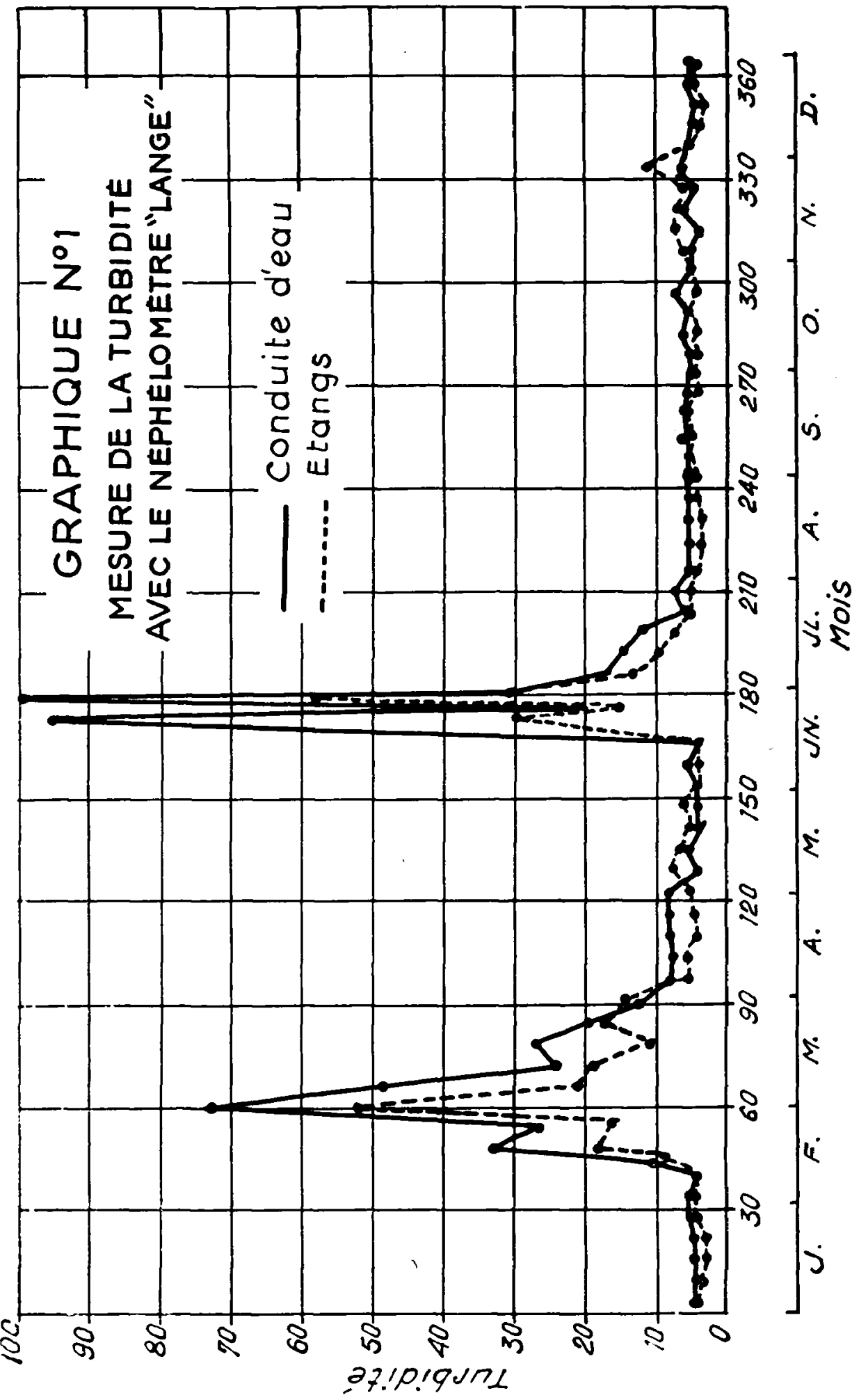


la végétation ne doit pas couvrir tout le fond du bassin; dans notre cas, il a suffit d'une surface de $40 \%$ du fond.

L'ensoleillement du bassin est favorable et indispensable pour cet élevage. Nous avons réalisé des expériences en mettant un bassin à l'ombre et, bien que la température de l'eau ait diminué sensiblement, l'oxygénation de cette eau était nettement inférieure à celle des autres bassins.

Finalement, en ce qui concerne le débit d'eau circulant dans les bassins, il faut signaler que celui-ci doit être beaucoup plus faible que dans les piscicultures en eaux froides. Bien que cela paraisse illogique au premier abord, c'est le moyen de permettre que les effets favorables obtenus par l'action des végétaux sursaturant l'eau en oxygène persistent dans le bassin.

Les résultats moyens obtenus par des dosages d'oxygène pratiqués hebdomadairement pendant les mois de juillet et août nous ont donné des valeurs variables selon l'influence de chacun des trois facteurs cités auparavant. On peut en détacher :

- En bassin élargi, pourvu de végétation avec un faible débit d'eau et exposé aux rayons solaires :

\begin{tabular}{|c|c|c|}
\hline \multicolumn{3}{|c|}{ Tableau 1} \\
\hline $\begin{array}{l}\text { Echantillon } \\
\text { prélevé à : }\end{array}$ & $\begin{array}{c}\text { Température } \\
\text { moyenne }\end{array}$ & $\mathrm{mg} \mathrm{O}_{2} /$ litre \\
\hline $6 \mathrm{~h} \ldots \ldots$ & $23^{\circ}$ & 8,5 \\
\hline $12 \mathrm{~h} \ldots \ldots \ldots$ & $24^{\circ}$ & 12,0 \\
\hline $18 \mathrm{~h} \ldots \ldots \ldots$ & $27^{\circ}$ & 17,4 \\
\hline
\end{tabular}

Bien que les températures atteintes, surtout le soir, étaient très élevées $\left(27^{\circ}\right)$, la tension d'oxygène dissous $\left(17,4 \mathrm{mg} \mathrm{O}_{2} / \mathrm{litre}\right)$ était supérieure de beaucoup aux $8-9 \mathrm{mg} \mathrm{O}_{2} /$ litre indispensables à l'espèce étudièe, et cela non seulement a permis son élevage mais nous attribuons sa grande activité physiologique à cette abondance d'oxygène. En même temps, cette activité a une plus grande durée pendant l'année à températures élevées que dans un élevage en eau froide.

En comparant deux bassins égaux, l'un étant exposé aux rayons solaires et l'autre protégé par un toit de roseaux pour qu'il reste à l'ombre, on observe que les quantités d'oxygène obtenues étaient les suivantes :

TABLEAU 2

\begin{tabular}{|c|c|c|c|}
\hline \multicolumn{2}{|l|}{$\begin{array}{l}\text { Échantillon } \\
\text { prélevé à : }\end{array}$} & Ensoleillé & A l'ombre \\
\hline & Température & $23^{\circ}$ & $23^{\circ}$ \\
\hline & $\mathrm{mg} \mathrm{O}_{2} /$ litre $\ldots . .$. & 7,5 & 7,0 \\
\hline $12 \mathrm{~h} . \ldots$ & Température ..... & $24^{\circ}$ & $24^{\circ}$ \\
\hline & $\mathrm{mg} \mathrm{O}_{2} /$ litre $\ldots \ldots$ & 10,5 & 8,0 \\
\hline $18 \mathrm{~h}$ & Température ..... & $26^{\circ}$ & $25^{\circ}$ \\
\hline & $\mathrm{mg} \mathrm{O}_{2} /$ litre . . & 14,0 & 8,5 \\
\hline
\end{tabular}


Bien que dans le bassin ensoleillé les températures de l'eau, comme il est logique étant donné la faible circulation, sont supérieures $\left(26^{\circ}\right)$ à celles du bassin à l'ombre $\left(25^{\circ}\right)$, cela entraîne que la tension d'oxygène est plus élevée (14 $\mathrm{mg} \mathrm{O}_{2} /$ litre) dans le premier cas que dans le second ( $8,5 \mathrm{mg} \mathrm{O}_{2}$ /litre) et ceci nous indique qu'il convient d'exposer les bassins aux rayons solaires. D'autre part, les individus en élevage se mettent à l'abri sous la végétation et ne sont pas exposés à un éclairage excessif.

\section{I. 4. - Température de l'eau.}

Dans les bassins où se pratiquait l'élevage de la Truite, on a relevé chaque jour les températures de l'eau à $9 \mathrm{~h}, 12 \mathrm{~h}$ et $18 \mathrm{~h}$.

Le tableau 3 donne les moyennes mensuelles correspondant aux années $1960,1961,1962,1963$ et 1964 . Dans ce même tableau nous voyons que les maxima des moyennes mensuelles correspondent aux mois de juillet et août pour lesquels on a atteint en 1961 à $18 \mathrm{~h}$, les températures de $26^{\circ}, 9$ et $26^{\circ}, 6$ respectivement.

\section{TABleEaU 3}

Température de l'eau des bassins en ${ }^{\circ} \mathrm{C}$, moyennes mensuelles.

\begin{tabular}{|c|c|c|c|c|c|c|c|c|c|c|c|c|c|}
\hline \multicolumn{2}{|c|}{ Années } & Janv. & Févr & Mars & Avril & M a i & Juin & Juil. & Août & Sept. & Oct. & Nov. & Déc. \\
\hline 1960. & $\left|\begin{array}{rl}9 & h \\
12 & h \\
18 & h\end{array}\right|$ & $\begin{array}{l}7,4 \\
8,1 \\
8,5\end{array}$ & $\begin{array}{l}9,0 \\
9,4 \\
9,5\end{array}$ & $\begin{array}{l}10,7 \\
11,3 \\
11,7\end{array}$ & $\begin{array}{l}13,4 \\
14,6 \\
15,3\end{array}$ & $\mid \begin{array}{l}17,5 \\
18,3 \\
19,2\end{array}$ & $\left|\begin{array}{l}22,1 \\
22,9 \\
24,2\end{array}\right|$ & $\left|\begin{array}{l}22,1 \\
24,0 \\
25,9\end{array}\right|$ & $\begin{array}{l}21,6 \\
23,4 \\
25,6\end{array}$ & $\begin{array}{l}19,6 \\
21,2 \\
21,5\end{array}$ & $\begin{array}{l}14,0 \\
14,5 \\
14,5\end{array}$ & $\begin{array}{l}11,0 \\
11,6 \\
11,6\end{array}$ & $\begin{array}{l}8,3 \\
9,0 \\
9,1\end{array}$ \\
\hline 1961. & $\begin{array}{rl}9 & h \\
12 & h \\
18 & h\end{array} \mid$ & $\begin{array}{l}6,7 \\
7,4 \\
7,9\end{array}$ & $\begin{array}{r}8,5 \\
9,8 \\
10,0\end{array}$ & $\begin{array}{l}11,0 \\
12,0 \\
12,3\end{array}$ & $\begin{array}{l}14,5 \\
15,3 \\
16,0\end{array}$ & $\left|\begin{array}{l}19,9 \\
22,2 \\
23,5\end{array}\right|$ & $\left|\begin{array}{l}20,0 \\
20,1 \\
23,7\end{array}\right|$ & $\left|\begin{array}{l}21,2 \\
25,1 \\
26,9\end{array}\right|$ & $\begin{array}{l}21,9 \\
24,1 \\
26,6\end{array}$ & $\begin{array}{l}19,9 \\
21,0 \\
21,4\end{array}$ & $\begin{array}{l}14,4 \\
16,0 \\
15,3\end{array}$ & $\begin{array}{l}11,4 \\
11,8 \\
11,8\end{array}$ & $\begin{array}{l}5,7 \\
6,6 \\
7,4\end{array}$ \\
\hline 1962. & $\left|\begin{array}{rr}9 & h \\
12 & h \\
18 & h\end{array}\right|$ & $\begin{array}{l}8,8 \\
9,3 \\
9,5\end{array}$ & $\begin{array}{l}6,9 \\
7,6 \\
8,6\end{array}$ & $\begin{array}{l}10,2 \\
10,8 \\
11,3\end{array}$ & $\begin{array}{l}13,3 \\
14,5 \\
15,1\end{array}$ & $\left|\begin{array}{l}17,8 \\
19,5 \\
20,3\end{array}\right|$ & $\left|\begin{array}{l}20,8 \\
22,5 \\
23,9\end{array}\right|$ & $\left|\begin{array}{l}22,9 \\
24,2 \\
26,3\end{array}\right|$ & $\begin{array}{l}21,6 \\
22,6 \\
24,0\end{array}$ & $\begin{array}{l}18,3 \\
19,1 \\
20,4\end{array}$ & $\begin{array}{l}15,8 \\
15,9 \\
16,5\end{array}$ & $\begin{array}{l}10,6 \\
10,7 \\
10,7\end{array}$ & $\begin{array}{r}9,1 \\
9,4 \\
10,0\end{array}$ \\
\hline 1963 & $\left|\begin{array}{rl}9 & h \\
12 & h \\
18 & h\end{array}\right|$ & $\begin{array}{l}8,4 \\
8,4 \\
8,5\end{array}$ & $\begin{array}{l}8,4 \\
8,4 \\
8,7\end{array}$ & $\begin{array}{l}11,4 \\
11,7 \\
12,4\end{array}$ & $\begin{array}{l}13,1 \\
14,1 \\
14,8\end{array}$ & $\mid \begin{array}{l}17,9 \\
19,4 \\
20,8\end{array}$ & $\left|\begin{array}{ll}21 & 2 \\
22 & , 0 \\
23 & , 5\end{array}\right|$ & $\mid \begin{array}{l}24,8 \\
25,5 \\
26,7\end{array}$ & $\begin{array}{l}22,9 \\
23,7 \\
25,4\end{array}$ & $\begin{array}{l}18,6 \\
19,4 \\
20,0\end{array}$ & $\begin{array}{l}15,5 \\
15,8 \\
16,6\end{array}$ & $\begin{array}{l}12,9 \\
12,9 \\
12,9\end{array}$ & $\begin{array}{l}7,8 \\
7,9 \\
8,1\end{array}$ \\
\hline 1964 & $\left|\begin{array}{rl}9 & h \\
12 & h \\
18 & h\end{array}\right|$ & $\begin{array}{l}7,2 \\
7,3 \\
8,0\end{array}$ & $\begin{array}{l}8,6 \\
9,0 \\
9,5\end{array}$ & $\begin{array}{l}11,6 \\
12,2 \\
13,3\end{array}$ & $\begin{array}{l}15,0 \\
15,6 \\
17,3\end{array}$ & $\left|\begin{array}{l}20,6 \\
21,6 \\
23,6\end{array}\right|$ & $\left|\begin{array}{l}21,4 \\
22,3 \\
23,5\end{array}\right|$ & $\mid \begin{array}{l}22,9 \\
24,0 \\
25,8\end{array}$ & $\begin{array}{l}21,4 \\
22,3 \\
23,9\end{array}$ & $\begin{array}{l}20,0 \\
20,9 \\
21,9\end{array}$ & $\begin{array}{l}13,9 \\
14,4 \\
15,1\end{array}$ & $\begin{array}{l}11,3 \\
11,3 \\
12,0\end{array}$ & $\begin{array}{r}8,8 \\
8,8 \\
10,0\end{array}$ \\
\hline
\end{tabular}

Dans le tableau 4 nous avons détaillé les températures maxima atteintes par l'eau dans les bassins chaque mois des mêmes années de 1960 à 1964 . Nous y voyons que $29^{\circ}$ a été le maximum pendant les mois de juillet et août et que les maxima des autres mois ne descendent pas 
au-dessous de $9^{\circ}$. Pour traiter d'une étude d'élevage en eaux à température très élevée nous avons souligné ces maxima.

\section{TABLEAU 4}

Températures maxima en ${ }^{\circ} \mathrm{C}$, relevées dans l'eau des bassins.

\begin{tabular}{|c|c|c|c|c|c|c|c|c|c|c|c|c|}
\hline Années & Janv. & Févr. & Mars & Avril & Mai & Juin & Juil. & Août & Sept. & oct. & Nov. & Déc. \\
\hline$\ldots \ldots \ldots \ldots$ & 11 & 13 & 13 & 17 & 23 & 26 & 27 & 27 & 25 & 19 & 13 & 9 \\
\hline 1961 & 9 & 11 & 14 & 21 & 27 & 26 & 29 & 29 & 23 & 17 & 15 & 12 \\
\hline 1962 & 12 & 10 & 14 & 20 & 23 & 27 & 27 & 28 & 22 & 18 & 13 & 10 \\
\hline 1963 & 10 & 10 & 14 & 20 & 22 & 28 & 28 & 27 & 23 & 20 & 15 & 12 \\
\hline 1964 & 9 & 13 & 17 & 22 & 26 & 26 & 28 & 27 & 24 & 20 & 14 & 11 \\
\hline
\end{tabular}

\section{2. Caractéristiques climatiques.}

\section{2. 1. - Température de l'air.}

Pendant l'année 1964, on a relevé chaque jour les températures maxima et minima de l'air dans la pisciculture, lesquelles étaient assez variables chaque mois ; c'est pourquoi dans le tableau 5 on a inclus outre les températures maxima et minima relevées chaque mois, la moyenne mensuelle des maxima et celle des minima.

\section{TABLEAU 5}

Températures maxima et minima de l'air, en ${ }^{\circ} \mathrm{C}$.

\begin{tabular}{|c|c|c|c|c|c|c|c|c|c|c|c|c|}
\hline & Janv. & Févr. & Mars & Avril & Mai & Juin & Juil. & Août & Sept. & Oct. & Nov. & Déc. \\
\hline Maxima ... & 14 & 15 & 22 & 29 & 36 & 41 & 39 & 38 & 34 & 26 & 17 & 11 \\
\hline $\begin{array}{c}\text { Moyenne mensuelle } \\
\text { des maxima }\end{array}$ & 8,8 & 11,5 & 15,8 & 20,1 & 31,7 & 32,0 & 35,5 & 33,1 & 28,3 & 18,8 & 12,8 & 8,4 \\
\hline Minima & $-\overline{\mathbf{5}}$ & -5 & -4 & 2 & 10 & 7 & 14 & 10 & 10 & 0 & -2 & -5 \\
\hline $\begin{array}{r}\text { Moyenne mensuelle } \\
\text { des minima } . . .\end{array}$ & $-1,2 \mid$ & 2,8 & 6,4 & 7,5 & 12,2 & 13,3 & 16,6 & 15,3 & 14,7 & 6,7 & 0,9 & $-0,6$ \\
\hline
\end{tabular}




\section{2. 2. - Ensoleillement.}

Comme l'oxygénation des eaux est en relation directe avec l'action des rayons solaires sur les plantes, nous indiquons les jours d'ensoleillement que nous avons eus chaque mois, de même que ces heures d'ensoleillement. Comme on peut le voir le plus grand nombre d'heures correspond aux mois de juillet et d'août, ce qui compense par son action bénéfique la température plus élevée de l'eau des bassins. Ces chiffres se rapportent à l'année 1962 et, en plus des données figurant dans le tableau 6 , nous devons signaler que le nombre total de jours de soleil pendant l'année a été de 341, que le nombre d'heures de soleil a été de $2.985,5$ et que la moyenne journalière d'heures pendant lesquelles le soleil a brillé a été de $8,45 \mathrm{~h}$.

Tableau 6

Jours et heures d'ensoleillement.

\begin{tabular}{|c|c|c|}
\hline Mois & Jours & Heures \\
\hline Janvier . . . . & 25 & 155,7 \\
\hline Février & 28 & 222,7 \\
\hline Mars .. & 25 & 141,8 \\
\hline$\ldots \ldots \ldots$ & 27 & 232,6 \\
\hline$\ldots \ldots$ & 31 & 318,8 \\
\hline Juin . & 30 & 330,5 \\
\hline Juillet & 31 & 419,7 \\
\hline Août $\ldots \ldots \ldots \ldots$ & 31 & 372,5 \\
\hline Septembre ...... & 30 & 271,6 \\
\hline Octobre $\ldots \ldots \ldots$ & 30 & 208,6 \\
\hline Novembre....... & 25 & 140,3 \\
\hline Décembre $\ldots \ldots$. & 28 & 171,7 \\
\hline
\end{tabular}

3. 3. Truite arc-en-ciel.

\section{3. I. - Incubation.}

L'àge des femelles reproductrices varie de deux ans (20 mois) à cinq ans (58 mois). Exceptionnellement on a laissé une femelle d'un an (11 mois) se reproduire.

Les mâles atteignent la maturité sexuelle la première année (10 mois) et dans de nombreux cas on les a utilisés pour la fécondation bien qu'en général on l'a effectuée avec des mâles de 2 ans (20 mois) et plus.

Le nombre de reproducteurs utilisés à chaque campagne a été assez élevé. Pour la frai de 1962-63, on a utilisé 143 mâles et 83 femelles, et pour celle de 1963-61, 125 mâles et 70 femelles.

Le nombre total d'œufs incubés a été de 124.383 pendant la campagne $1962-63$ et 155.121 pendant celle de $1963-64$. 
Les résultats qui figurent dans les différents groupes d'incubation se rapportent toujours aux chiffres moyens obtenus en travaillant avec le total de reproducteurs et d'œufs sus mentionné et, plus précisément, pendant les campagnes $1962-63$ et $1963-64$.

\section{3. 1. 1. - Epoque de frai.}

L'époque de reproduction a varié entre le 27 novembre et le 2 mars, présentant sa fréquence maximum entre la $2^{\mathrm{e}}$ quinzaine de décembre et la $1^{\text {re }}$ quinzaine de février. La durée de la période de frai a été de 62 jours pendant la campagne de $1962-63$ et de 83 jours pendant celle de $1963-64$.

\section{3. 1. 2. - Temperature d'incubation.}

La température d'incubation a été relevée deux fois par jour et les moyennes sont celles qui figurent dans le calcul de degrés-jours.

Les moyennes hebdomadaires de la température correspondant à la période d'incubation de l'année 1963-64 apparaissent dans le graphique $n^{\circ} 2$, n'ayant pas tracé les courbes correspondant aux autres années parce qu'elles présentaient des variations peu notables par rapport à la précédente.

La température moyenne d'incubation de $1962-63$ a varié entre $8^{\circ}, 1$ et 9०,97, ce dernier chiffre correspondant aux pontes effectuées en février. Les températures moyennes les plus fréquentes se situent entre $8^{\circ}, 3$ et $8^{\circ}, 5$.

Les températures moyennes correspondant à la campagne 1963-64 ont varié entre $7^{\circ}, 97$ et $10^{\circ}, 90$, les plus élevées correspondant aussi aux pontes effectuées en février, mais les valeurs les plus fréquentes se situant entre $8^{\circ}, 1$ et $8^{\circ}, 6$.

La moyenne des températures moyennes en 1962-63 a été de $8^{\circ}, 5$ et pendant $1963-64$ de $8^{\circ}, 9$.

Aussi, nous pouvons remarquer que la température minimum de l'eau pendant l'incubation $1962-63$ a été de $6^{\circ}$ et de $7^{\circ}$ en $1963-64$, alors que la température maximum atteinte pendant l'incubation en 1962-63 a été de $13^{\circ}$ et de $12^{\circ}$ en 1963-64.

\section{3. I. 3. - Eufs embryonnes.}

Le total de degrés-jours qui ont été nécessaires aux œufs fécondés jusqu'à l'apparition des yeux (œufs embryonnés) a varié pendant 1962-63 entre 250 et 210 degrés-jours, ces chiffres correspondant respectivement à des reproductions effectuées les premiers jours de décembre et les premiers jours de février. Les chiffres les plus fréquents ont oscillé entre 222 et 228 degrés-jours et la moyenne relative à toutes les reproductions effectuées a été de 223 degrés-jours.

Pendant la campagne 1963-64, les valeurs extrêmes ont été 239 et 217 degrés-jours. Affectant aussi le maximum à des reproductions pré- 
$-65-$

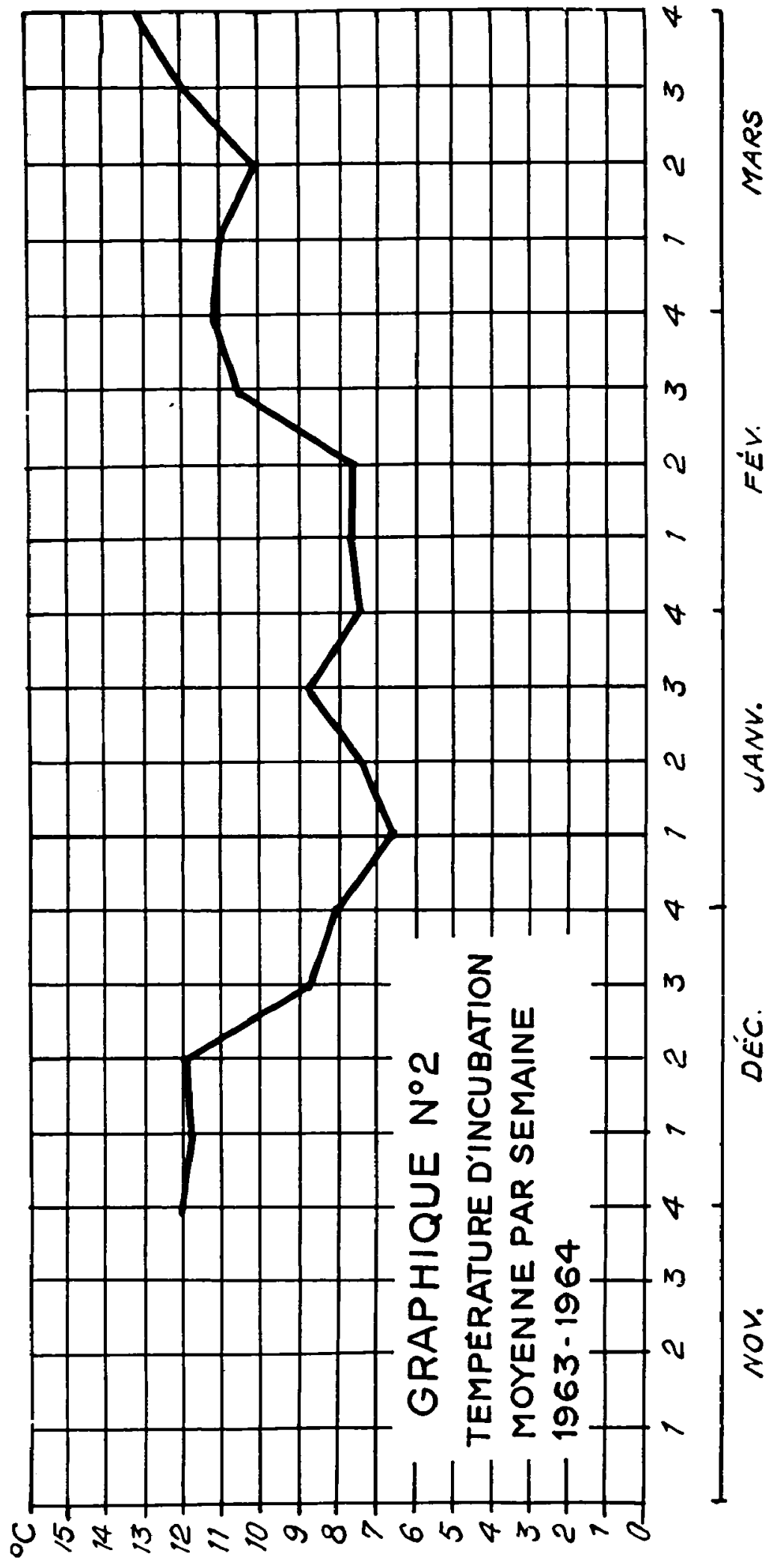


coces effectuées les derniers jours de novemgre, la moyenne de degrésjours pour atteindre le stade d'œufs embryonnés a été de 229 pendant cette période.

Le nombre de jours écoulés depuis la fécondation jusqu'à l'apparition des yeux pendant $1962-63$ a varié entre 32 et 23 jours, la plus grande fréquence se situant entre 25 et 28 jours.

Pendant 1963-64, il a varié entre 30 et 20 jours, la plus grande fréquence se situant entre 23 et 28 jours.

La moyenne des jours écoulés entre la fécondation et l'apparition des yeux en $1962-63$ a été de 26,6 jours et 25,8 jours en $1963-64$.

Le pourcentage de pertes pendant le stade étudié, à savoir de la fécondation à l'apparition des yeux, a atteint une valeur moyenne de 7,72 en $1962-63$ et de 8,60 en 1963-64. Nous devons signaler qu'il y a eu quelques cas isolés de pertes extraordinaires que nous avons écartés à cause de leur faible fréquence ou d'anomalies de la femelle ou de la distribution d'eau dans le bac d'incubation par suite d'avaries ou d'autres causes.

\section{3. 1. 4. Eclosion.}

Le total de degrés-jours qu'il a fallu aux œufs fécondés pour éclore a varié entre 336 et 412 pendant la campagne 1962-63. Les valeurs les plus fréquentes ont varié entre 350 et 356 , et la moyenne relative à toutes les incubations effectuées a été de 371. Pendant la campagne 1963-64, les valeurs extrêmes ont été 299 et 374 degrés-jours. Les valeurs les plus fréquentes ont varié entre 343 et 357 et la moyenne de cette campagne pour atteindre l'éclosion a été de 347 degrés-jours.

Le nombre de jours écoulés depuis la fécondation jusqu'à l'éclosion a varié entre 38 et 47 pendant la campagne 1962-63, la plus grande fréquence se situant entre 41 et 44 jours, et la valeur moyenne a été de 43,6 jours.

Pendant la campagne 1963-64, il a varié entre 32 et 47 jours et les valeurs les plus fréquentes ont varié entre 39 et 44 jours, la valeur moyenne étant de 39,8 jours.

En relation avec le total d'œufs morts pendant l'incubation comprenant, par conséquent, les pertes subies jusqu'à l'apparition des yeux,de l'apparition des yeux à l'éclosion, et pendant l'éclosion, les pourcentages correspondants pendant la campagne 1962-63 atteignent une valeur moyenne de $20,45 \%$ et de $27,67 \%$ pendant $1963-1964$.

\section{3. 2. - Alevinage.}

\section{3. 2. I. - Résorption de la vésicule.}

Le total de jours écoulés de l'éclosion jusqu'à la résorption de la vésicule vitelline a varié pendant 1962-63 entre 22 et 40 jours avec une valeur moyenne de 30,9 .

Durant 1963-64, le nombre de jours a varié entre 19 et 39 jours, avec une moyenne de 27,8 jours. 
Les pertes totales pendant la résorption de la vésicule ont atteint une moyenne de $9,12 \%$ pendant $1962-63$, et $11,32 \%$ pendant $1963-64$.

\section{3. 2. 2. - Alevins jusqu'd l'age de 3 mois (en bacs).}

Bien que normalement dans la pisciculture les alevins après la résorption de la vésicule restent en bacs pendant une courte durée d'environ un mois et demi, pour les expériences rapportées dans ce chapitre, les alevins sont restés dans les bacs pendant 3 mois.

Les bacs utilisés mesuraient $2 \times 0,45 \times 0,49 \mathrm{~m}$ et dans chacun on a mis 2.250 alevins, ce qui donne une densité de 2.500 alevins au mètre carré. Tous les alevins provenaient de la même fécondation effectuée le 2 décembre 1963 et étaient nés le 14-1-1964. Ils ont été transférés des bacs où ils avaient effectué la résorption de la vésicule dans ceux indiqués plus haut le 10-2-1964, date à laquelle cette étude a été commencée.

Les uns ont été nourris avec de la rate et les autres avec du plancton. Tous les 10 jours on a contrôlé les longueurs et les poids atteints, en prenant 5 individus et en calculant les moyennes correspondantes.

Dans les tableaux 7 et 8 et les graphiques 3 et 4 , on notera que les dates de départ correspondent à un âge de 30 jours qui était celui des alevins à cette date.

\section{TABLEAU 7}

\begin{tabular}{||c|c|c|c|c|c|c|c|c|c|c||}
\hline \hline $\begin{array}{c}\text { Nourri- } \\
\text { ture }\end{array}$ & $\begin{array}{c}\text { Age } \\
\text { (en jours) }\end{array}$ & 30 & 40 & 50 & 60 & 70 & 80 & 90 & 100 & 110 \\
\hline Rate & $\begin{array}{r}\text { Longueur } \\
\text { (en mm) }\end{array}$ & 24,0 & 26,4 & 27,2 & 32,2 & 37,0 & 41,2 & 44,2 & 45,0 & 51,2 \\
\hline $\begin{array}{r}\text { Poids (en } \\
\text { g) ... }\end{array}$ & 0,09 & 0,11 & 0,15 & 0,27 & 0,37 & 0,68 & 0,93 & 0,99 & 1,58 \\
\hline $\begin{array}{r}\text { Longue ur } \\
\text { (en mm) }\end{array}$ & 24,4 & 25,0 & 26,0 & 27,0 & 29,2 & 31,2 & 37,2 & 38,0 & 42,6 \\
\hline $\begin{array}{r}\text { Poids (en } \\
\text { g) .... }\end{array}$ & 0,09 & 0,09 & 0,11 & 0,13 & 0,19 & 0,27 & 0,41 & 0,33 & 0,70 \\
\hline
\end{tabular}

Les alevins morts pendant l'expérience étaient ceux qui figurent dans le tableau suivant :

\section{Tableau 8}

Pourcentage des pertes.

\begin{tabular}{lccc} 
& \multicolumn{3}{c}{ Age (en jours) } \\
Nourriture & $30-60$ & $60-90$ & $90-120$ \\
Rate . . . . . & - & - & - \\
Plancton . . & 8,13 & 2,80 & 3,77 \\
& 8,80 & 15,55 & 16,80
\end{tabular}



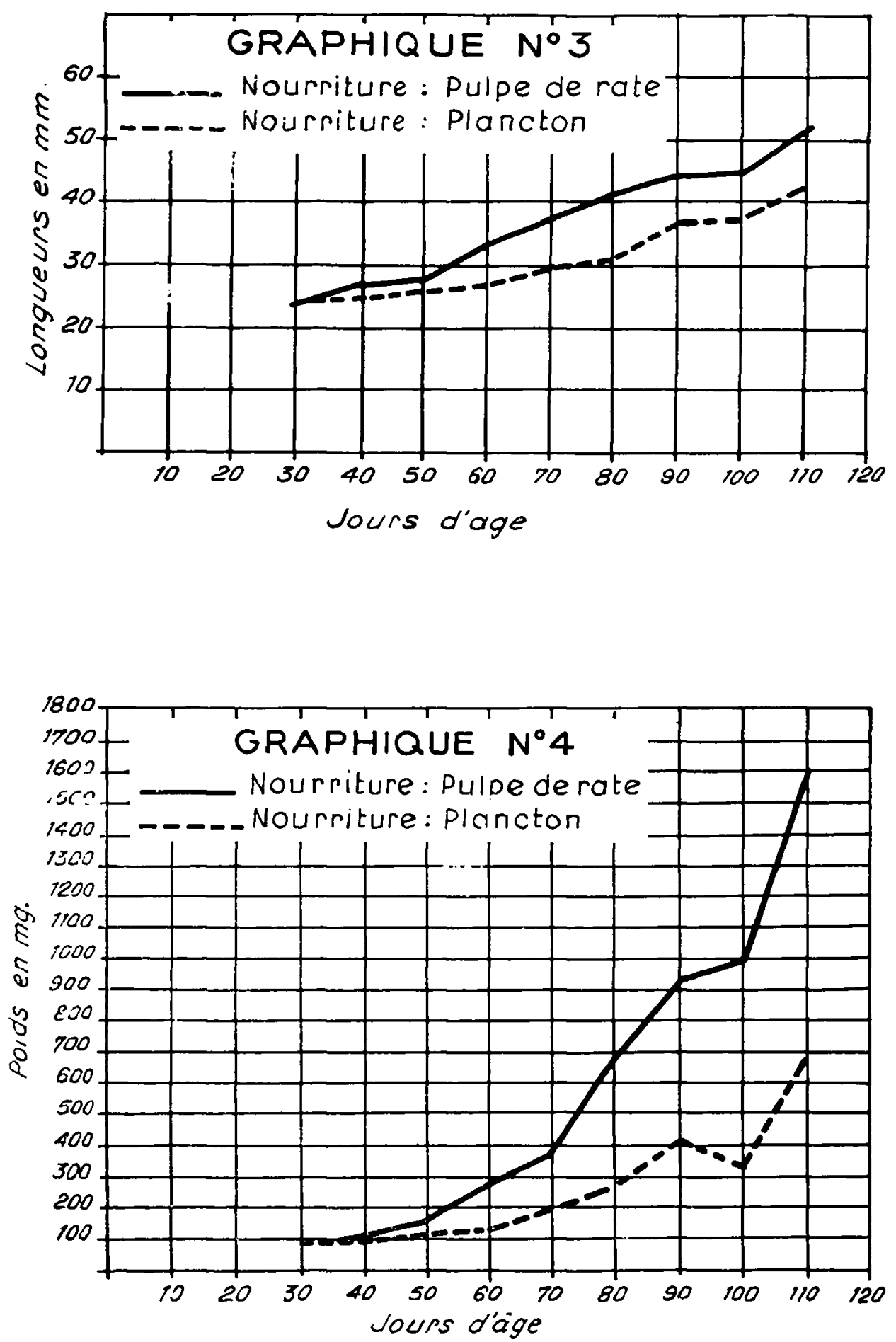
Relevons que l'alimentation avec la rate est plus favorable, mais nous croyons qu'elle a été favorisée du fait que le plancton qui a été donné en nourriture n'était pas assez abondant et que le courant d'eau qui circulait dans les bacs l'entrainait vers le trop-plein où il se perdait.

Nous avons observé, au contraire, de bons rendements en poids et en taille avec des alevins alimentés avec du plancton en aquarium. Nous pouvons aussi signaler, outre cette expérience concrète, que normalement dans la pisciculture, pendant la période située entre la résorption et la libération des alevins dans les bassins, ils sont soumis à un régime de rate enrichie avec du plancton composé de daphnies élevé dans la pisciculture même et les résultats ainsi obtenus sont meilleurs. 